\title{
Análisis multicriterio de plantas medicinales cultivadas en la vereda Ceylan del municipio de Viotá, Cundinamarca, Colombia
}

\author{
Multi-criteria Analysis of the Medicinal Plants Grown in \\ Ceylan, municipality of Viotá, Cundinamarca, Colombia
}

\section{Yuri Alicia Chávez-Plazas ${ }^{\text {ac }}$, María Lucero Ramírez-Mahecha ${ }^{\text {ad }}$, Judith Elena Camacho-Kurmen ${ }^{\text {be }}$}

\author{
a Grupo de investigación ODISEA, Facultad Ciencias Sociales, Universidad Colegio Mayor de Cundinamarca, Colombia \\ ${ }^{\mathrm{b}}$ Grupo de investigación Bioprocesos y Control (GBYC), Facultad Ciencias de la Salud, Universidad Colegio Mayor de Cundinamarca, Colombia \\ 'yachavez@unicolmayor.edu.co | https://orcid.org/0000-0003-0115-7010 \\ d mluceroramirez@unicolmayor.edu.co|https://orcid.org/0000-0002-3378-6033 \\ e jelenacamacho@unicolmayor.edu.co | https://orcid.org/0000-0002-8880-1501
}

\section{RESUMEN}

El presente trabajo se orientó a realizar el análisis multicriterio de plantas medicinales cultivadas en la vereda Altos de Ceylan, municipio de Viotá (Cundinamarca, Colombia), como un paso hacia la certificación ecológica y el aprovechamiento sostenible de la biodiversidad existente en el país, combinados con el diálogo de saberes, con el fin de rescatar el conocimiento tradicional de la comunidad y construir nuevos conocimientos. El análisis multicriterio define los criterios ambientales y económicos que se ponderaron para la selección de las plantas medicinales a certificar como productos ecológicos. "El diálogo de saberes" es una metodología que establece un intercambio de saberes entre la academia - en este caso la Universidad Colegio Mayor de Cundinamarca y la Universidad Nacional de Colombia-, los investigadores y los cono-

Citation: Chávez-Plazas, Y. A., Ramírez-Mahecha, M. C. y Camacho-Kurmen, J. E. (2021). Análisis multicriterio de plantas medicinales cultivadas en la vereda Ceylan del municipio de Viotá, Cundinamarca, Colombia. Mutis, 11(1), 22-36. https://doi.org/10.21789/22561498.1751

Recibido: Abril 6, 2021. Aceptado: Mayo 13, 2021.

Copyright: @2021. Chávez-Plazas, Y. A., Ramírez-Mahecha, M. C. y Camacho-Kurmen, J. E. (2021). This is an open-access article, which permits unrestricted use, distributions and reproduction in any medium, provided the original author and source are credited. cimientos de las comunidades locales, representadas en esta investigación por las mujeres rurales pertenecientes a la Asociación Semillas de Esperanza y Paz de Mujeres Víctimas del Conflicto Armado (Asepamuvic). El conocimiento construido versó sobre los saberes tradicionales con respecto al cultivo y el manejo y uso de plantas medicinales y aromáticas cultivadas en la vereda, identificando como especies promisorias a la sábila (Aloe vera Linneo), el limoncillo (Cymbopogon citratus), el prontoalivio (Lippia alba-Verbenaceae), la manzanilla (Matricaria chamomilla Linneo), la

Competing Interests: The authors have no conflict of interest. 
hierbabuena (Mentha piperita Linneo), el tomillo (Pectis graveolens Klatt), el perejil (Petroselinum crispum (Mill.)) y el romero (Rosmarinus officinalis Linneo).

Palabras clave: biodiversidad, economía ambiental, intercambio de conocimientos, medicina tradicional.

\section{ABSTRACT}

This work carried out a multi-criteria analysis of the medicinal plants grown in the village "Altos de Ceylan," municipality of Viotá (Cundinamarca, Colombia), as a step towards the ecological certification and sustainable use of the existing plant biodiversity in Colombia, combined with the dialogue of knowledge, which is aimed at rescuing traditional knowledge by local communities and building new insights. The multi-criteria analysis allowed us to define the environmental and economic criteria weighted for the selection of the medicinal plants to be certified as ecological products. "The dialogue of knowledge" is a methodology that establishes an exchange of knowledge between the academy - in this case, Universidad Colegio Mayor de Cundinamarca and the National University of Colombia-, researchers and local communities, represented in this study by women belonging to "Seeds of Hope and Peace, Association of Women Victims of Armed Conflict" [Asociación Semillas de Esperanza y Paz de Mujeres Víctimas del Conflicto Armado] (Asepamuvic). The insights built encompassed traditional knowledge regarding the cultivation, management and use of locally-grown medicinal and aromatic plants, making it possible to identify promising species such as sábila (Aloe vera Linnaeus), lemongrass (Cymbopogon citratus), prontoalivio (Lippia alba-Verbena), chamomile (Matricaria chamomilla Linneo), mint (Mentha piperita Linneo), thyme (Pectis graveolens Klatt), parsley (Petroselinum crispum (Mill.)), and rosemary (Rosmarinus officinalis Linneo), based on the analysis of environmental and economic criteria.

Keywords: Biodiversity, environmental economics, knowledge exchange, traditional medicine.

\section{INTRODUCCIÓN}

La Organización Mundial de la Salud (OMS) ha defendido la utilidad y conservación de los saberes tradicionales desde la medicina tradicional, formulando periódicamente, desde 2002, los lineamientos estratégicos de medicina tradicional para el mundo. Este ente internacional resalta además la importancia de conservar plantas medicinales para su uso desde la práctica de atención prioritaria en salud, como quedó establecido en la Declaración de Chiang Mai de 1988 (OMS, 1988). Por lo anterior, este trabajo se llevó a cabo de manera conjunta con las comunidades rurales de la vereda de Altos Ceylan, particularmente con la Asociación Semillas de Esperanza y Paz de Mujeres Víctimas del Conflicto Armado (Asepamuvic), desde lo estipulado en la Política Nacional Para la Gestión Integral de la Biodiversidad y sus Servicios Ecosistémicos, donde se establece la importancia de conocer, preservar, restaurar, conservar y usar sosteniblemente la biodiversidad para identificar especies promisorias de plantas nativas (MADS, 2012).
El diálogo de saberes hace parte de la metodología de investigación-acción participativa (IAP), la cual se basa en la interacción entre distintos actores para el reconocimiento de los problemas propios de su territorio u organización. Este abordaje permite plantear alternativas de mejoramiento al problema estudiado, para lo cual se requiere de la participación de la comunidad y de la generación de aprendizaje mutuo durante todo el proceso (Delgado \& Rist, 2016; Ochoa, 2012).

El diálogo de saberes es importante para encontrar puntos en común que permitan validar y fortalecer la experiencia de los actores y los espacios que estos intervienen, con el fin de consolidar un marco conceptual a partir de los encuentros, las reflexiones y las estrategias de estos actores para atender las necesidades, los acuerdos y los enfoques establecidos, acogiendo así el llamado de la interculturalidad como proceso vigente dentro del desarrollo de los estudios sociales y comunitarios (Delgado \& Rist, 2016; Ochoa, 2012; Romero \& Rodríguez, 2010). Adicionalmente, desde otra perspectiva, el diálogo intercultural permite que las comunidades se sientan respetadas e involucradas en el tránsito a través del conocimiento continuo de la enfermedad 
hacia la salud (Delgado \& Rist, 2016; Hernández-Rincón et al., 2017; Ochoa, 2012).

De acuerdo con cifras reportadas por el Departamento Administrativo Nacional de Estadística (DANE), solo durante 2017 las ventas al exterior de hierbas aromáticas lograron un crecimiento de $18 \%$, alcanzando un total de USD 37,2 millones (La República, 2018). Además, en 2018 Colombia exportó 101.054 toneladas de plantas medicinales y aromáticas, siendo Estados Unidos, Canadá y la Unión Europea los principales destinos. Las variedades que más se venden al extranjero son albahaca, hierbabuena, tomillo y romero (MADR, 2019).

Los productos naturales y las plantas medicinales en Colombia cuentan con estándares de calidad que los garantizan, además de la vigilancia del Ministerio de Salud y el Instituto Nacional de Vigilancia en Medicamentos y Alimentos (Invima), siendo este último el encargado de otorgar los registros sanitarios, establecer las buenas prácticas de manufactura y regular la comercialización y producción de este tipo de productos por medio de la "lista básica", en la cual se encuentran las plantas medicinales aptas para su uso y consumo, siendo estas en mayor proporción especies no nativas (Castellanos et al., 2017; Invima, 2019).

El uso comercial de plantas medicinales genera beneficios monetarios y no monetarios, dentro de los cuales se cuentan el conocimiento, la conservación y el uso sostenible de especies promisorias, tanto a nivel medicinal como industrial, la creciente tendencia del mercado a exigir calidad en las materias primas y la eficiencia en el proceso productivo para la obtención de la planta medicinal en términos de volumen, continuidad y calidad. Además de lo anterior, se tienen como beneficios el valor agregado que se genera al recuperar conocimientos tradicionales y utilizar la bioprospección; la capacitación que se brinda a cultivadores, recolectores y compradores, resaltando la importancia de los valores locales e individuales, así como el valor del medio ambiente; y la generación y el establecimiento de negocios viables y sustentables, que hacen posible generar desarrollo local, regional y nacional a través del uso comercial de plantas medicinales de manera sustentable (Kate \& Laird, 2019).

Con base en lo anterior, esta investigación tiene como objetivo realizar un análisis multicriterio (AMC) en el que se ponderan las categorías y los criterios de selección ambientales y económicos establecidos por
Camacho y Castiblanco (2008) para establecer las plantas medicinales y aromáticas a incorporar dentro del proceso de certificación ecológica de las especies cultivadas en la vereda Altos de Ceylan. Para ello, este trabajo adopta diferentes estrategias formuladas desde el diálogo de saberes, las cuales permiten la integración del conocimiento popular y científico (Reyes-Sánchez, 2009). El AMC propuesto se basa en la ponderación y compensación de variables, ya sean determinantes o factores de aptitud, exigiendo la indicación de pesos o ponderaciones para cada factor (Sipahi \& Mehpare, 2010).

\section{MATERIALES Y MÉTODOS}

El desarrollo de esta investigación acerca de la aplicación de AMC para el proceso de certificación de plantas medicinales y aromáticas, que se da en el contexto del proyecto "Diálogo de saberes: una experiencia investigativa con mujeres rurales de Viotá orientada a la certificación ecológica en plantas medicinales y aromáticas", tiene en cuenta las fases del proceso de diálogo de saberes desarrollado con mujeres rurales de Viotá asociadas a Asepamuvic, para lo cual se aplicó la metodología propuesta por Morse (1994), cuyas fases serán descritas a continuación.

\section{Fase de reflexión}

Esta fase consiste en la identificación del tema, la pregunta de investigación y la perspectiva metodológica. Durante esta fase se utilizó la metodología del tendedero social, la cual es ampliamente usada en entornos colaborativos. Esta metodología proporciona un espacio físico para que los participantes presenten sus resultados (escritos o dibujados), después de la realización de las dinámicas propuestas, con el fin de generar un registro visible de lo que sucede en una comunidad (BBVA, 2017). Se realizaron talleres de capacitación e intercambio de experiencias con 15 mujeres rurales integradas a Asepamuvic y, eventualmente, dos hombres de la vereda, teniendo en cuenta el conocimiento tradicional de los participantes y el conocimiento científico construido en la universidad referente a formas de cultivo. Esto permitió identificar los usos de las plantas, las medidas de bioseguridad requeridas para su manejo y las buenas prácticas recomendadas para su cultivo. Así mismo, fue posible resolver preguntas tales como ¿qué plantas reconozco?, 
¿qué usos y beneficios tienen estas?, ¿cuáles son los métodos de cultivo tradicionales y ecológicos?, ¿cuál sería la viabilidad económica de tales métodos?

\section{Fase de planificación}

En esta fase se llevó a cabo la selección del contexto, las estrategias y la propuesta de investigación (visitas previas, reconocimiento del contexto y de la población). Como estrategia, se trabajó por grupos, donde cada uno de estos estableció las plantas aromáticas que cultivaría y la técnica de cultivo a emplear a partir de la construcción de un protocolo de cultivo. El objetivo de esta fase fue significar el conocimiento de las comunidades y complementarlo con el conocimiento construido desde la universidad. Esta primera actividad finalizó con una visita a los cultivos tradicionales de los participantes.

\section{Fase de entrada}

Se seleccionaron los informantes y los casos. De igual forma, se inició la realización de entrevistas y observaciones (planificación de los posibles escenarios de los diálogos). Además, se elaboraron herbarios con las plantas medicinales y aromáticas cultivadas en la vereda, en los que se incluyeron el nombre de cada planta, sus usos y preparaciones. Lo anterior tuvo como objetivo rescatar el conocimiento tradicional sobre las plantas en la vereda Altos de Ceylan. El trabajo se desarrolló en tres etapas: proyecto productivo, trabajo en equipo y aprovechamiento de la biodiversidad con respecto a plantas medicinales y aromáticas (conocer, conservar, restaurar y utilizar) (MADS, 2012).

La muestra de participantes estuvo conformada por 13 mujeres y 2 hombres residentes de la vereda, en edades comprendidas entre 18 y 75 años. Varios de los participantes se dedican al cultivo de frutales y café (Senna hirsuta), así como a la cría y venta de porcinos. La mayoría de estos cuenta con nivel de educación primario, mientras que un pequeño grupo no ha recibido educación formal. Los participantes emplean sus huertas caseras para el cultivo de plantas medicinales.

\section{Fase de recolección reproductiva de la información}

En esta etapa se realizó una primera devolución de la información construida mediante el diálogo de saberes a los participantes. Para ello, se retomó la información de los herbarios, la cual fue complementada con información científica ampliada frente a los usos de cada planta, su nombre científico, usos y propiedades. Esto permitió reforzar el importante recurso que significa cada planta identificada.

\section{Fase de salida de campo}

Esta fase se realizó en tres momentos. En primer lugar, se visitaron los cultivos de plantas medicinales en las huertas de los participantes. El segundo momento contempló la realización de una exposición sobre las características del cultivo y la aplicación de buenas prácticas agrícolas (BPA) por parte de cada grupo de participantes, actividad que fue retroalimentada por los investigadores. El tercer momento fue la visita del Instituto Agroecológico María Cano al proyecto, la cual tuvo como objetivo fortalecer la elaboración de productos derivados de las plantas cultivadas y seleccionadas.

\section{Análisis multicriterio}

Se utilizó la metodología de análisis multicriterio (AMC) desarrollada por Camacho y Castiblanco (2008) para ponderar y seleccionar las plantas medicinales que serían incluidas en el programa de certificación ecológica, luego de comprobar que dichas plantas fueron cultivadas de manera orgánica, sin el uso de agroquímicos (MADR, 2019).

\section{Diseño experimental y análisis de datos}

Se realizó un análisis multicriterio en el que se asignó un valor a los criterios ambientales y económicos considerados, como se muestra a continuación.

\section{Criterio ambiental}

\section{Especies nativas}

En Colombia, el universo de plantas con actividad medicinal es amplio (156 especies comercializadas). Por ende, se tuvo en cuenta el criterio de que la planta medicinal fuese una especie nativa para así aprovechar la biodiversidad del país. Además, se consideró la frecuencia de comercialización de dicha especie, así como la demanda de los laboratorios naturistas y su inclusión en el listado de plantas medicinales y aromáticas comercializadas en Colombia (Bernal et al., 2011; 
Castellanos et al., 2017; Invima, 2019; Rincón-Parra, 2012). Así, se consideran los siguientes criterios:

- Criterio: nativa $=100 \%$.

- Criterio: no nativa $=0 \%$ (naturalizada [adaptada a nuestros ecosistemas], foránea o importada).

\section{Seguridad del uso de la especie (calidad, efectividad e inocuidad)}

Para cada una de las especies seleccionadas se realizó un estudio bibliográfico tomando en cuenta aspectos relacionados con la seguridad del uso de la especie, lo cual hace referencia a que sean plantas efectivas (con acción farmacológica), que cumplan con las normas de calidad de las farmacopeas y que sean inocuas, es decir, sin antecedentes de toxicidad. Al respecto, se tiene los siguientes criterios:

- Estudios farmacológicos, toxicológicos y clíni$\cos =50 \%$.

- Inclusión en lista básica del Invima = $50 \%$.

- No se conocen estudios $=0 \%$.

- No inclusión en la lista básica del Invima = 0 \%

\section{Aprovechamiento de la biodiversidad}

En lo relacionado con el aprovechamiento de la biodiversidad existente, se cuentan los siguientes criterios:

- Participación en estudios de bioprospección $=50 \%$.

- Conocimiento tradicional documentado $=50 \%$.

- Sin información = 0 \%.

Para seleccionar los criterios antes descritos se toma como referente el objetivo principal de la Política Nacional de Biodiversidad, que es conocer, conservar y hacer uso sostenible de la biodiversidad a través de acciones como recuperar y divulgar el conocimiento y las prácticas tradicionales y hacer uso de la bioprospección para financiar la conservación de la biodiversidad (MADS, 2012).

\section{Fuentes, sustentabilidad y calidad de la materia prima}

La sustentabilidad se relaciona con el número de especies promisorias y la forma en la que se obtiene la planta medicinal (recolección silvestre o cultivo). Como criterio se tiene:

- Mayor sustentabilidad $=10$ puntos.

- Más o menos sustentable $=5$ puntos

- Menor posibilidad de sustentabilidad $=2$ puntos.

La manera en la que se obtiene la planta medicinal tiene implicaciones importantes para la sustentabilidad del proceso, por lo que es importante diferenciar si la obtención de la planta se hace por recolección silvestre o por cultivo, puesto que a partir del método de obtención se ven afectados factores como la utilidad, la fluctuación de oferta, el control de calidad, la identificación botánica, el mejoramiento genético, la manipulación agronómica, el manejo postcosecha y la posible adulteración de una especie determinada (Kate \& Laird, 2019).

\section{Criterios económicos}

\section{Valor económico total (VET)}

Para este análisis se tuvo en cuenta el concepto de valor económico total (VET) planteado por David Pearce (Pearce \& Turner, 1995), el cual hace referencia a todos los posibles valores de un bien ambiental, independientemente de que estos tengan precios de mercado o no.

Desde esta perspectiva, se consideran los valores de uso (directo e indirecto) y de no uso (valor de opción y valor de existencia) de las plantas medicinales, puesto que la contribución porcentual de las especies y los ecosistemas silvestres a la economía de los países en desarrollo, como Colombia, suele ser mucho mayor, por lo cual se toma como referente la mencionada valoración (tabla 1). 
Tabla 1. Categorías de valor incorporadas al análisis de valor económico total de los bienes de un ecosistema

\begin{tabular}{|c|c|c|c|}
\hline \multicolumn{2}{|c|}{ Valor de uso } & \multicolumn{2}{|c|}{ Valor de no uso } \\
\hline Valor de uso directo & Valor de uso indirecto & Valor de opción & Valor de existencia \\
\hline Alimentos vegetales $=1$ & Retención de sedimentos $=1$ & Especies $=1$ & Especies en extinción $=1$ \\
\hline Farmacéuticos $=1$ & Retención nutrientes = 1 & Conservación de hábitat = 1 & Estética = 1 \\
\hline Materia prima $=1$ & Soporte a biodiversidad = 1 & Protección de la biodiversidad = 1 & Conservación = 1 \\
\hline Investigación = 1 & Producción de $\mathrm{O}_{2}=1$ & Potencial farmacéutico $=1$ & - \\
\hline Reproducción de especies = 1 & Secuestro $\mathrm{CO}_{2}=1$ & - & - \\
\hline Biomasa $=1$ & Belleza escénica = 1 & - & - \\
\hline Plantas medicinales $=1$ & Polinización = 1 & - & - \\
\hline Plantas ornamentales $=1$ & Reproducción de especies $=1$ & - & - \\
\hline Subtotal $=8$ & Subtotal $=8$ & Subtotal $=4$ & Subtotal $=3$ \\
\hline
\end{tabular}

Fuente: Barzev (2002).

Como criterio, se toma cada categoría como variable dicotómica, de la siguiente manera:

- No aporta al valor $=0$.

- Aporta al valor $=1$.

- Valor total (valor de uso + valor de no uso $)=23$.

Muchos ecosistemas naturales reportan beneficios de carácter indirecto y su valor económico deriva de los servicios que proveen, más que de los productos que se pueden obtener de ellos. Por ende, se tuvo en cuenta tanto el valor de uso como el de no uso, ya que, por ejemplo, muchas plantas están dotadas de atributos socioculturales y religiosos que les otorgan una condición especial. Por su parte, el estudio del carácter físico o biológico de las plantas ha redundado en la acumulación de conocimientos científicos básicos de utilidad, que a su vez ha sentado las bases para nuevos adelantos y nuevos descubrimientos (Jiménez-Herrero, 1997).

\section{Otros criterios económicos}

Dentro de este conjunto de criterios se tiene en cuenta el valor agregado derivado de la recuperación del conocimiento tradicional y su utilización en la bioprospección. Así mismo, se incluyen la capacitación a cultivadores, recolectores y compradores - proceso en el que se resaltan los valores locales, individuales y del medio ambiente- $\mathrm{y}$ la generación y el establecimiento de negocios viables y sustentables, con lo cual se genera desarrollo local, regional y nacional a partir del uso comercial de plantas medicinales desde una perspectiva sustentable en términos económicos, sociales y ecológicos.

Dentro del grupo de otros criterios económicos se incluyeron las especies promisorias, las necesidades del mercado, la eficiencia, el valor agregado, el desarrollo local y la capacitación, asignado un máximo de 10 puntos por categoría.

\section{Necesidades del mercado}

Este criterio hace referencia a la calidad del producto. Al ser plantas ampliamente comercializadas en Colombia, y con potencial a nivel internacional, surge la necesidad de incluir esta variable, puesto que a partir de criterios de calidad y del conocimiento de la especie medicinal correcta será posible cubrir el mercado local en cuanto a volumen (cantidades), eventualmente abriendo paso a la posibilidad de exportar estos productos.

\section{Eficiencia}

La sostenibilidad (eficiencia) se logra cuando se cuenta con cosechas productivas, se conservan los recursos (especies, biodiversidad, agua y suelo), se satisfacen las necesidades económicas y sociales, se evidencian sistemas viables de producción, se valora 
el conocimiento tradicional y la información y se registran bajos niveles de plagas y enfermedades. Otros determinantes de la eficiencia son la educación, la investigación, la difusión y el desarrollo de la agricultura internacional.

\section{Valor agregado}

Para determinar el valor agregado de los productos se tiene en cuenta que estos sean cosechados por comunidades locales, aprovechando el conocimiento y las capacidades de la mujer, quien tiene un papel primordial en el cultivo, las familias y la comunidad, generando así beneficios sociales, empleo y una economía de subsistencia para la región. Además, en esta dimensión se valora el conocimiento tradicional asociado al desarrollo y los métodos tradicionales de cultivo, así como los estudios de investigación y de bioprospección realizados.

\section{Capacitación y desarrollo local}

Se debe tener en cuenta la importancia de la capacitación en buenas prácticas agrícolas y de recolección para todos los actores que intervienen en el proceso de producción de plantas medicinales, situación que redundará en desarrollo local y regional en forma sostenible.

\section{RESULTADOS}

Los participantes de esta investigación pertenecen a la asociación Asepamuvic. La edad promedio de los participantes fue 36 años. El $98 \%$ de los asistentes son mujeres y el $2 \%$ restante corresponde a hombres. El nivel de escolaridad de los participantes está entre educación primaria y secundaria (67\%), técnica (13\%) y universitaria (7\%), encontrando una tasa de analfabetismo de $13 \%$. El $4 \%$ de los participantes son adultos mayores con gran conocimiento tradicional sobre el uso de plantas medicinales y aromáticas.

El listado de plantas medicinales que se encuentran presentes y se cultivan en la vereda Altos de Ceylan, Viotá, Cundinamarca, se construyó teniendo en cuenta el nombre tradicional y científico de cada una de ellas, así como sus usos, preparaciones y propiedades. Las plantas incluidas en este listado son: limonaria (Cymbopogon citratus), hierba del dolor (Acalypha arvensis), poleo (Minthostachys tomentosa), lengua de suegra (Sansevieria trifasciata Prain), perejil
(Petroselinum crispum (Mill.)), borrachero (Atropa beIladonna Linneo), guaba (Phytolacca icosandra), comfrey (Symphytum officinale Linneo), sauco (Sambucus nigra Linneo), ruda (Ruta graveolens Linneo), sábila (Aloe vera Linneo), caléndula (Calendula officinalis Linneo), romero (Rosmarinus officinalis Linneo), apio (Apium graveolens Linneo), manzanilla (Matricaria chamomilla Linneo), hierbabuena (Mentha piperita Linneo), orégano (Origanum vulgare Linneo), menta (Menta piperita Linneo subsp. citrata (Ehrh Briq.)), toronjil (Melissa officinalis Linneo), mirto (Psychotria rufescens), altamisa (Ambrosia arborescens Mill.), tomillo (Pectis graveolens Klatt), paico (Chenopodium ambrosioides), albahaca (Ocimun basilicum Linneo), amápola (Papaver rhoeas Linneo), prontoalivio (Lippia alba-Verbenaceae), cayeno (Hibiscus rosa-sinensis) y ají (Capsicum annuum Linneo var. minimum (Mill.)).

Como complemento, se realizó un intercambio de saberes entre el conocimiento tradicional y el científico con base en la literatura consultada (AlarcónRestrepo, 2011; Bernal et al., 2011; Castellanos et al., 2017; Duque, 2002; ICA, 2015; Invima, 2019; García et al., 2016; Ministerio de la Protección Social, 2008), lo que permitió la selección de las plantas promisorias.

Por otra parte, se compartió con los participantes el estudio realizado por Córdoba (2017), en el que se presenta un plan de negocios para la creación de la comercializadora de hierbas aromáticas orgánicas "Sabor de Origen". Este trabajo destaca a la albahaca (Ocimun basilicum Linneo) (81,25\%), el tomillo (Pectis graveolens Klatt) $(67,5 \%)$, el laurel (65\%), el orégano (60\%), el romero (Rosmarinus officinalis Linneo) $(28,75 \%)$ y el estragón (12,5 \%) como las especies de mayor interés para la comercialización a escala internacional, gracias a las preferencias de los consumidores. Como se observa en el listado de plantas de la vereda Altos de Ceylan, algunas de estas especies se cultivan allí.

De otro lado, el $90 \%$ de las personas encuestadas por Córdoba (2017) manifiesta que actualmente compra hierbas aromáticas valorando la frescura del producto, resultado que puede ser aprovechado por los participantes para la selección de las plantas y el proyecto productivo a desarrollar en la vereda. Este hallazgo es respaldado por el Instituto Colombiano Agropecuario (ICA, 2015), que destaca la comercialización y exportación en fresco de plantas medicinales y aromáticas. 


\section{Cultivo plantas medicinales}

Los integrantes de Asepamuvic trabajaron en tres parcelas cultivando romero (Rosmarinus officinalis Linneo), manzanilla (Matricaria chamomilla Linneo), hierbabuena (Mentha piperita Linneo) y sábila (Aloe vera Linneo), actividad para la que adoptaron las BPA recomendadas por la OMS (2003) y además tuvieron en cuenta las condiciones ambientales de la vereda, las características del suelo, la disponibilidad de agua y el tipo de semillas a utilizar. Al tratarse de cultivos ecológicos, se descartó el uso de plaguicidas de origen químico para las labores de manejo agronómico (Amaya et al., 2008).

\section{Análisis multicriterio}

Para el cumplimiento de esta actividad se utilizó el listado de plantas medicinales y aromáticas consideradas como relevantes para los propósitos de este proyecto, el cual fue construido de manera conjunta con los participantes. La realización del AMC consideró las categorías ambientales y económicas y los criterios empleados para su valoración, como se detalla a continuación.

\section{Categoría ambiental}

\section{Especies nativas}

La tabla 2 muestra el detalle de las especies nativas y no nativas identificadas en el sitio estudiado, incluyendo la relación de sus nombres científicos.

Tabla 2. Especies nativas y no nativas

\begin{tabular}{|c|c|c|c|}
\hline \multirow{2}{*}{ Planta } & \multirow{2}{*}{ Nombre científico } & \multicolumn{2}{|c|}{ Origen } \\
\cline { 3 - 4 } & Nativa & $\begin{array}{c}\text { No } \\
\text { nativa }\end{array}$ \\
\hline Apio & Apium graveolens Linneo & 0 & 100 \\
\hline Ají & $\begin{array}{c}\text { Capsicum annuum Linneo } \\
\text { var. minimum (Mill.) }\end{array}$ & 0 & 100 \\
\hline Amapola & Papaver rhoeas Linneo & 0 & 100 \\
\hline Albahaca & Ocimun basilicum Linneo & 0 & 100 \\
\hline Altamisa & Ambrosia arborescens Mill & 0 & 100 \\
\hline $\begin{array}{c}\text { Borrachero } \\
\text { (Belladona) }\end{array}$ & Atropa belladonna Linneo & 0 & 100 \\
\hline Caléndula & Calendula officinalis Linneo & 0 & 100 \\
\hline
\end{tabular}

\begin{tabular}{|c|c|c|c|}
\hline Cayena & Hibiscus rosa-sinensis & 0 & 100 \\
\hline Comfrey & $\begin{array}{c}\text { Symphytum officinale } \\
\text { Linneo }\end{array}$ & 0 & 100 \\
\hline Guaba & Phytolacca icosandra & 0 & 100 \\
\hline Hierba del dolor & Acalypha arvensis & & \\
\hline Hierbabuena & Mentha piperita Linneo & 0 & 100 \\
\hline $\begin{array}{l}\text { Lengua de suegra } \\
\text { milagrosa }\end{array}$ & Sansevieria trifasciata Prain & 0 & 100 \\
\hline $\begin{array}{l}\text { Limonaria o } \\
\text { limoncillo }\end{array}$ & Cymbopogon citratus & 0 & 100 \\
\hline Manzanilla & $\begin{array}{l}\text { Matricaria chamomilla } \\
\text { Linneo }\end{array}$ & 0 & 100 \\
\hline Menta & $\begin{array}{c}\text { Menta piperita Linneo } \\
\text { subsp. citrata (Ehrh) Briq. }\end{array}$ & 0 & 100 \\
\hline Mirto & Psychotria rufescens & 0 & 100 \\
\hline Orégano & Origanum vulgare Linneo & 0 & 100 \\
\hline Paico & Chenopodium ambrosioides & 100 & 0 \\
\hline Perejil & Petroselinum crispum (Mill.) & 100 & 0 \\
\hline Poleo & Minthostachys tomentosa & 0 & 100 \\
\hline Prontoalivio & Lippia alba-Verbenaceae & 100 & 0 \\
\hline Ruda & Ruta graveolens Linneo & 0 & 100 \\
\hline Romero & $\begin{array}{l}\text { Rosmarinus officinalis } \\
\text { Linneo }\end{array}$ & 0 & 100 \\
\hline Sábila & Aloe vera Linneo & 0 & 100 \\
\hline Sauco & Sambucus nigra Linneo & 0 & 100 \\
\hline Tomillo & Pectis graveolens Klatt & 100 & 0 \\
\hline Toronjil & Melissa officinalis Linneo & 0 & 100 \\
\hline
\end{tabular}

Fuente: elaboración propia con base en información de Bernal et al. (2011).

\section{Seguridad del uso de la especie (calidad, efectividad e inocuidad), fuentes, sustentabilidad y calidad de la materia prima}

La tabla 3 presenta el estudio bibliográfico realizado a las plantas medicinales. Para ello se tomaron en cuenta los aspectos relacionados con la seguridad del uso de la especie mencionados anteriormente. Además, se revisaron los estudios de bioprospección realizados, el conocimiento tradicional documentado y la sustentabilidad de cada especie, dimensión relacionada con el número de especies promisorias y la manera en que se obtiene la planta medicinal (recolección silvestre o cultivo). 
Tabla 3. Seguridad del uso de la especie, aprovechamiento de la biodiversidad y sustentabilidad

\begin{tabular}{|c|c|c|c|c|c|c|}
\hline Planta & $\begin{array}{l}\text { Seguridad del uso de la } \\
\text { especie (estudios farma- } \\
\text { cológicos y toxicológicos) }\end{array}$ & $\begin{array}{c}\text { Seguridad del uso de la } \\
\text { especie (inclusión lista } \\
\text { Invima) }\end{array}$ & $\begin{array}{l}\text { Estudios de biopros- } \\
\text { pección }\end{array}$ & $\begin{array}{l}\text { Conocimiento } \\
\text { tradicional docu- } \\
\text { mentado }\end{array}$ & Sustentabilidad & Total \\
\hline Apio & 50 & 50 & 50 & 50 & 10 & 210 \\
\hline Ají & 50 & 50 & 50 & 50 & 10 & 210 \\
\hline Amapola & 50 & 0 & 50 & 50 & 2 & 152 \\
\hline Albahaca & 50 & 50 & 50 & 50 & 10 & 210 \\
\hline Altamisa & 50 & 0 & 50 & 50 & 2 & 152 \\
\hline Borrachero (Belladona) & 50 & 50 & 50 & 50 & 5 & 205 \\
\hline Caléndula & 50 & 50 & 50 & 50 & 10 & 210 \\
\hline Cayena & 50 & 0 & 50 & 50 & 5 & 205 \\
\hline Consuelda (Comfrey) & 50 & 0 & 50 & 50 & 5 & 155 \\
\hline Guaba & 50 & 50 & 50 & 50 & 10 & 210 \\
\hline Hierba del dolor & 50 & 0 & 50 & 50 & 2 & 152 \\
\hline Hierbabuena & 50 & 50 & 50 & 50 & 10 & 210 \\
\hline $\begin{array}{l}\text { Lengua de suegra mila- } \\
\text { grosa }\end{array}$ & 50 & 0 & 50 & 50 & 5 & 155 \\
\hline Limonaria o limoncillo & 50 & 50 & 50 & 50 & 10 & 210 \\
\hline Manzanilla & 50 & 50 & 50 & 50 & 10 & 210 \\
\hline Menta & 50 & 0 & 50 & 50 & 5 & 155 \\
\hline Mirto & 50 & 0 & 50 & 50 & 5 & 155 \\
\hline Orégano & 50 & 50 & 50 & 50 & 10 & 210 \\
\hline Ortiga & 50 & 50 & 50 & 50 & 10 & 210 \\
\hline Paico & 50 & 0 & 50 & 50 & 5 & 155 \\
\hline Perejil & 50 & 50 & 50 & 50 & 10 & 210 \\
\hline Poleo & 50 & 0 & 50 & 50 & 2 & 152 \\
\hline Prontoalivio & 50 & 50 & 50 & 50 & 10 & 210 \\
\hline Ruda & 50 & 50 & 50 & 50 & 5 & 205 \\
\hline Romero & 50 & 50 & 50 & 50 & 10 & 210 \\
\hline Sábila & 50 & 50 & 50 & 50 & 10 & 210 \\
\hline Sauco & 50 & 50 & 50 & 50 & 10 & 210 \\
\hline Tomillo & 50 & 0 & 50 & 50 & 10 & 210 \\
\hline Toronjil & 50 & 50 & 50 & 50 & 5 & 205 \\
\hline
\end{tabular}

Fuente: elaboración propia. 


\section{Categoría económica}

\section{Valor económico total (VET)}

La utilización del concepto de VET dentro de los criterios de selección se debe a que este permite valorar en su totalidad el aporte de las plantas medicinales al valor económico total de los servicios ambientales de un ecosistema, permitiendo conocer el valor del bien en sí y de sus servicios y funciones como parte de un ecosistema (Barzev, 2002; Camacho \& Lancheros, 2008; Pearce \& Turner, 1995). El análisis correspondiente se representa en la figura 1.

Figura 1. Valor económico total de las especies estudiadas

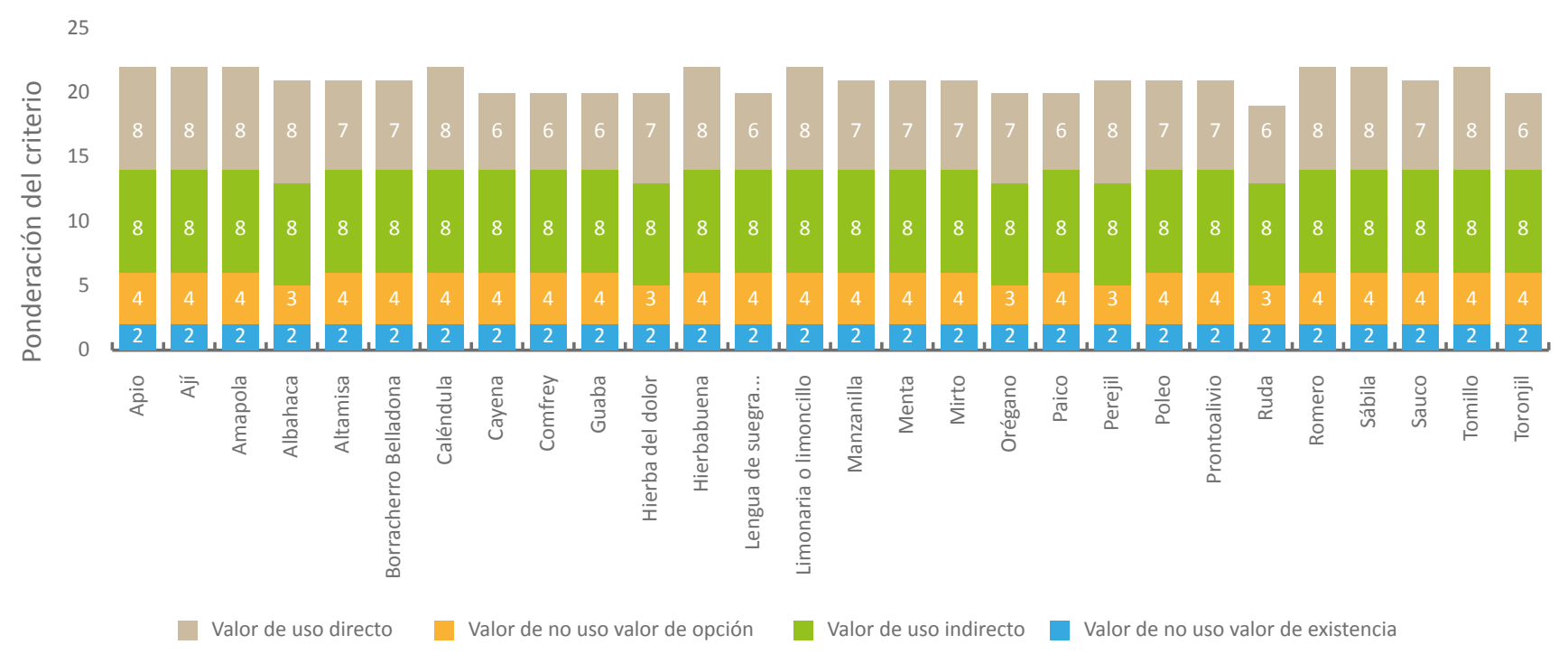

Fuente: elaboración propia.

\section{Especies promisorias para uso comercial}

Se ha establecido que algunas plantas de especies diferentes pueden tener el mismo nombre común dependiendo de las ciudades donde se comercialicen. Sin embargo, no se cuenta con información precisa sobre el volumen de las diferentes especies de plantas consumidas o comercializadas (Díaz, 2003).

El criterio ambiental toma factores como la seguridad de uso de la planta, el aprovechamiento de la biodiversidad y la fuente, y la sustentabilidad y calidad de la materia prima, lo cual nos orientó a la selección de las plantas medicinales sábila, limoncillo, prontoalivio, manzanilla, hierbabuena, tomillo, perejil y romero.

En el criterio económico se tiene como referencia el valor de uso y el valor de no uso, seleccionando las mismas plantas mencionadas en el criterio ambiental debido a su aporte significativo al valor de no uso (valor de opción y valor por existencia), producto de su potencial farmacéutico e industrial. Desde el valor de uso, estas especies cuentan con diversos usos indirectos que dan soporte al ecosistema y, así mismo, contribuyen al valor de uso directo como plantas medicinales, materias primas, alimentos y plantas ornamentales. Además, estas plantas no corresponden a cultivos extensivos, poseen aplicaciones generalmente específicas e incluyen un aporte considerable de valor agregado en conocimiento tradicional y mano de obra, lo cual se enriquece con procesos de investigación, bioprospección y capacitación desarrollados para cultivos ecológicos, aportando así al desarrollo local, regional y nacional a través de la educación y la gestión ambiental (García et al., 2016), como se muestra en la figura 2. 
Figura 2. Categorías económicas

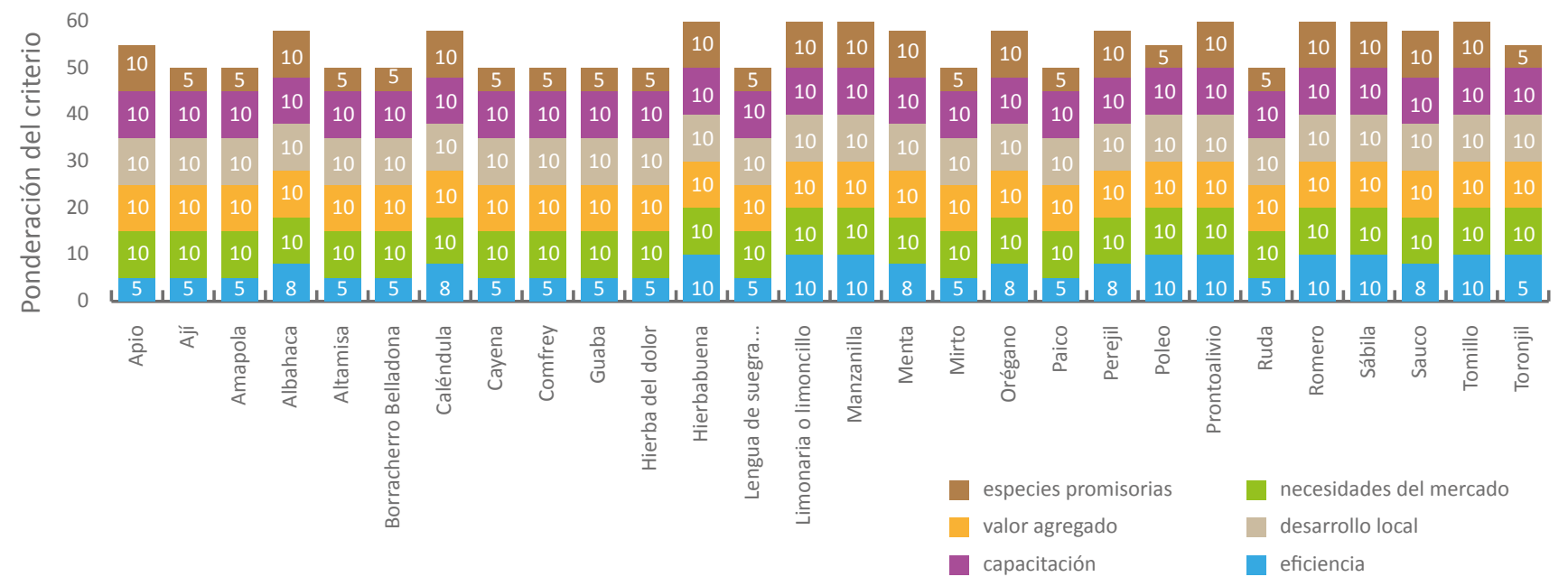

Fuente: elaboración propia.

\section{DISCUSIÓN}

El análisis multicriterio aplicado a la ponderación de los criterios ambientales y económicos necesarios para seleccionar un grupo de plantas de interés a ser involucradas en procesos productivos y programas de certificación ecológica, teniendo en cuenta la política Nacional de Biodiversidad y sus principios (conocer, conservar, restaurar y utilizar) (MADS, 2012), acudió a la apropiación social del conocimiento a través del diálogo de saberes. En lo ambiental, se dio prioridad al origen nativo de la especie seleccionada para aprovechar la biodiversidad de Colombia en términos de plantas medicinales, así como la bioprospección y el conocimiento tradicional como valor agregado, sumados al concepto de valor económico total (VET) (Pearce \& Turner, 1995).

Las plantas medicinales seleccionadas son recolectadas en su mayoría en forma silvestre, sin control alguno o la realización de capacitaciones para los recolectores, quienes en su mayoría se han dedicado a esta labor por años. Durante el proceso se evidencia la falta de cumplimiento de pautas elementales como recolectar las plantas en el momento adecuado de su desarrollo vegetal o no llevar a cabo esta labor cerca de carreteras o zonas industriales. También se da cuenta de un número importante de personas involucradas en el cultivo de estas especies que no tiene experiencia o capacitación alguna, lo que influye en el impacto ecológico de esta actividad agrícola y a su vez difunde un manejo inadecuado para la obtención de plantas de uso medicinal de calidad. Esto resulta importante debido a que en los últimos años la demanda de plantas medicinales se ha multiplicado y la población rural o citadina acude a este tipo de actividades para subsistir (Kate \& Laird, 2019).

Como parte del trabajo desarrollado en la vereda Altos de Ceylan se construyó una matriz aprender-desaprender sobre plantas medicinales y aromáticas, similar a lo propuesto por autores como Gallegos-Zurita (2016), quien considera a las plantas medicinales como la principal alternativa para el cuidado de la salud. Además, esta investigación permitió conocer métodos de cultivo basados en el conocimiento tradicional, la aplicación de BPA (OMS, 2003), el manejo de la gestión ambiental y el control de plaguicidas químicos (Amaya et al., 2008) mediante el uso alternativo de bioplaguicidas (Camacho et al., 2010).

Los participantes del estudio construyeron herbarios y apreciaron su propio saber al evidenciar que este era valorado y que sus opiniones eran tomadas en cuenta para la toma de decisiones. Estas estrategias han sido desplegadas por autores como Sandoval (2018), quien muestra la importancia de los procesos educativos participativos y horizontales basados en saberes 
y prácticas locales en asociaciones campesinas como Agruco, donde se resalta la agricultura biológica, sana y limpia para la conservación del medio ambiente y el fortalecimiento de las bases productivas, mejorando la calidad de vida de las familias campesinas. El estudio realizado con Asepamuvic hizo posible romper asimetrías, especialmente aquellas entre los saberes campesinos y los saberes técnicos, puesto que ambas se lograron fusionar para la construcción de miradas colectivas integrales, como se aborda en estudios similares (García-de-Alba et al., 2012; Leff et al., 2004; Rivera et al., 2017; Sandoval, 2018).

Se enunciaron las especies de plantas medicinales identificadas tal y como se denominan localmente, complementando esta información con el saber científico dado por el Invima (2019), el Instituto de Investigación de Recursos Biológicos Alexander von Humboldt (Bernal et al., 2011) y el ICA (AlarcónRestrepo, 2011; ICA, 2015). Por lo anterior, se recomienda realizar una identificación certificada de estas especies a fin de llevar su trazabilidad y poder establecer aquellas que se encuentran y se cultivan en la vereda, como fue hecho por Vera y Sánchez (2015), quienes registraron algunas plantas medicinales cultivadas en el corregimiento de San Cristóbal, municipio de Medellín.

Con esta selección se pretende mostrar el potencial de la biodiversidad de nuestro país con respecto a las variedades comercializadas en otros países y llamar la atención hacia variedades nativas aún no explotadas, estimulando su cultivo y permitiendo cumplir la posible demanda de especies promisorias como el prontoalivio (Lippia alba-Verbenaceae) y el perejil (Petroselinum crispum (Mill.)).

Para esta investigación se diseñaron talleres de capacitación con los miembros de la asociación Asepamuvic orientados a establecer la estrategia pedagógica, la metodología de trabajo, las técnicas y los contenidos a abordar. De esta manera, se generaron diversos materiales impresos que contribuyeron a que los participantes pudieran profundizar en el abordaje de las distintas temáticas tratadas. Esto concuerda con lo trabajado por Rivera (2017), quien brindó espacios de diálogo donde los participantes compartieron sus problemas, experiencias y expectativas sobre el manejo de la vida silvestre, aprendiendo con ello sobre el manejo técnico de esta actividad.
Las plantas medicinales tienen como beneficios monetarios el incremento de precios, avances en pagos para la realización de estudios de investigación, pagos por propiedad intelectual y otros beneficios financieros (investigación + desarrollo). Como beneficios no monetarios se resalta la provisión de infraestructura, equipos, tecnologías y asistencia a programas enfocados a estandarizar los sistemas médicos tradicionales, así como el mejoramiento del manejo de especies nativas. Actualmente, el mercado de este tipo de productos recibe apoyo institucional para el proceso de certificación ecológica por parte de la Federación Nacional de Cafeteros, Cenicafé, la Corporación Colombia Internacional, el Consejo de Administración Forestal (FSC), Biointropic, el Departamento de Agricultura de los Estados Unidos (USDA) y EurepgAP. Por ello, se espera lograr dicha certificación e incrementar la competitividad de estos productos tanto en el entorno nacional como en los mercados internacionales (MADR, 2019).

En cuanto a los participantes del proyecto, se rescata que los tiempos de las comunidades están basados en ciclos naturales y políticos relacionados con la dinámica de sus comunidades, donde la prioridad es el futuro inmediato de sus familias, la productividad de sus actividades y el beneficio de la comunidad. Estos factores fueron mencionados por los participantes como motivaciones para continuar haciendo parte de este tipo de iniciativas, lo cual es particularmente relevante al considerar la participación de las mujeres rurales, que en este trabajo fue bastante destacada.

\section{CONCLUSIONES}

El uso del análisis multicriterio y el diálogo de saberes permitió enriquecer el trabajo realizado con Asepamuvic frente a la utilidad y la valoración ambiental y económica de las plantas aromáticas y medicinales presentes y cultivadas en la vereda Altos de Ceylan, ubicada en el municipio de Viotá (Cundinamarca, Colombia), como parte de un proceso productivo.

De acuerdo con el AMC efectuado, las plantas seleccionadas para la siembra de cultivos y su inclusión en programas de certificación ecológica son sábila (Aloe vera Linneo), limoncillo (Cymbopogon citratus), prontoalivio (Lippia alba-Verbenaceae), manzanilla (Matricaria chamomilla Linneo), hierbabuena (Mentha piperita Linneo), tomillo (Pectis graveolens 
Klatt), perejil (Petroselinum crispum (Mill.)) y romero (Rosmarinus officinalis Linneo). Dentro de este grupo se destacan el prontoalivio y el perejil, plantas nativas que se encuentran en el listado del Invima (2019).

\section{AGRADECIMIENTOS}

Las autoras agradecen a los miembros de Asepamuvic por su participación entusiasta en el desarrollo de este trabajo de investigación. También agradecen a la Universidad Colegio Mayor de Cundinamarca por el apoyo recibido durante su realización.

\section{REFERENCIAS}

Alarcón-Restrepo, J. J. (2011). Plantas aromáticas y medicinales: enfermedades de importancia y usos terapéuticos. Medidas para la temporada invernal. ICA.

Amaya, E. F., Roa, A. M., Camacho, J. E., \& Meneses, S. (2008). Valoración de factores de riesgo asociados a los hábitos de manejo y exposición a organofosforados y carbamatos en habitantes y trabajadores de la vereda de Bateas del municipio de Tibacuy, Cundinamarca, Colombia. Nova, 6(10), 147-155. https://doi.org/10.22490/24629448.405

Barzev, R. E. (2002). Guía metodológica de valoración económica de bienes, servicios e impactos ambientales. Un aporte para la gestión de ecosistemas y recursos naturales en el CBM. Corredor Biológico Mesoamericano.

BBVA (2017, agosto 8). Descubre las tecnologías sociales para entornos colaborativos. https://www. bbva.com/es/descubre-tecnologias-sociales-entornos-colaborativos/

Bernal, H. Y., García-Martínez, H., \& QuevedoSánchez, G. F. (2011). Pautas para el conocimiento, conservación y uso sostenible de las plantas medicinales nativas en Colombia: Estrategia nacional para la conservación de plantas. Instituto de Investigación de Recursos Biológicos Alexander von Humboldt.
Camacho, J. E., \& Castiblanco, C. (2008). Certificación ecológica de dos plantas medicinales como un paso hacia el aprovechamiento de la biodiversidad en Colombia. Medio Ambiente y Desarrollo. Aporte de los estudiantes del PMAD al debate ambiental. Universidad Nacional de Colombia.

Camacho, J. E., Gómez, M. I., \& Villamizar, L. F. (2010). Selección de un sistema de atomización para la formación de micropartículas de eudragit ${ }^{\circledR}$ s100 en lecho fluido. Nova, 8(13), 87-100. https:// doi.org/10.22490/24629448.442

Castellanos-Castro, C., Sofrony, C., \& Higuera, D. (eds.). (2017). Plan de acción de la Estrategia Nacional para la Conservación de Plantas de Colombia. Instituto de Investigación de Recursos Biológicos Alexander von Humboldt, Ministerio de Ambiente y Desarrollo Sostenible, \& Red Nacional de Jardines Botánicos de Colombia. https://doi.org/10.21068/B001.2016.304

Córdoba, R. (2017). Plan de negocio para la creación de la comercializadora de hierbas aromáticas orgánicas - Sabor de Origen (tesis de maestría, Universidad Santo Tomás). Repositorio USTA. https://repository.usta.edu.co/ handle/11634/9784

Delgado, F., \& Rist, S. (eds.). (2016). Ciencias, diálogo de saberes y transdisciplinariedad. Agroecología Universidad Cochabamba.

Diaz, J. (2003). Informe Técnico. Caracterización del Mercado Colombiano de Plantas Medicinales y Aromáticas. I. Instituto de Investigación de Recursos Biológicos Alexander von Humboldt.

Duque, A. (2002). Informe del primer seminario iberoamericano de comercialización de plantas medicinales y aromáticas. Boletín Latinoamericano y del Caribe de plantas Medicinales y Aromáticas, 1(2), 12-14. https:// www.redalyc.org/pdf/856/85610205.pdf

Gallegos-Zurita, M. (2016). Las plantas medicinales: principal alternativa para el cuidado de la salud, en la población rural de Babahoyo, Ecuador. Anales de la Facultad de Medicina 77(4), 327-332. https://doi.org/10.15381/anales. v77i4.12647 
García-de-Alba, J. E., Ramírez-Hernández, B. C., Robles-Arellano, G., Zañudo-Hernández, J., Salcedo-Rocha, A. L., \& García-de-Alba-Verduzco, J. E. (2012). Conocimiento y uso de las plantas medicinales en la zona metropolitana de Guadalajara. Desacatos, 39, 29-44. https://doi. org/10.29340/39.238

García-Martínez, H., Moreno, L. A., Londoño, C., \& Sofrony, C. (2016). Estrategia nacional para la conservación de plantas: actualización de los antecedentes normativos y políticos y revisión de avances. Instituto de Investigación de Recursos Biológicos Alexander von Humboldt.

Hernández-Rincón, E. H., Lamus-Lemus, F., CarrataláMunuera, C., \& Orozco-Beltrán, D. (2017). Diálogo de saberes: propuesta para identificar, comprender y abordar temas críticos de la salud de la población. Revista Salud Uninorte, 33(2), 242-251. https://doi.org/10.14482/sun.33.2.10552

Instituto Colombiano Agropecuario [ICA]. (2015, octubre 20). Predios productores de frutales, hortalizas y hierbas aromáticas registrados en el ICA para exportación en fresco. https://www.ica. gov.co/icacomunica/prensa/el-registro-de-predios-productores-agropecuarios-f

Instituto Nacional de Vigilancia de Medicamentos y Alimentos [Invima]. (2018). Listado de plantas medicinales aceptadas con fines terapéuticos. https://www.invima.gov.co/documents/20143/461703/ LISTADO-DE-PLANTAS-JULIO-31-2018. pdf/7bdad908-d5d8-76aa-c2bf-41590611066a

Jiménez-Herrero, L. M. (1997). Desarrollo sostenible y economía ecológica. Síntesis.

Kate, K., \& Laird, S. A. (2019). The commercial use of biodiversity: Access to genetic resources and benefit-sharing. Routledge. https://doi. org/10.4324/9780429341540

La República (2018) La producción de los cultivos de plantas aromáticas y especias crecieron $21 \%$ en 2017. https://www.larepublica.co/economia/ laproduccion-de-los-cultivos-de-plantas-aromaticas-y-especias-crecieron-21-en2017-2771263
Leff, E. (2004). Racionalidad ambiental y diálogo de saberes. Significancia y sentido en la construcción de un futuro sustentable. Polis. Revista Latinoamericana, 2(7), 13-40. https://doi.org/10.5380/dma.v7i0.3042

Ministerio de Agricultura y Desarrollo Rural [MADR]. (2019). Lista de organismos de certificación acreditados y autorizados para certificar bajo el reglamento para la producción ecológica adoptado por la Resolución 0187/2006, y autorizados para administrar el uso del Sello de Alimento Ecológico. MADR https://www.minagricultura. gov.co/tramites-servicios/Documents/Lista_de_ Certificadoras_de_Productos_Ecologicos_040219. pdf

Ministerio de Agricultura y Desarrollo Rural [MADR]. (2019). Cadena de plantas aromáticas, medicinales, condimentarías y afines. Dirección de cadenas agrícolas y forestales. MADR.

Ministerio de Ambiente y Desarrollo Sostenible [MADS]. (2012). Política Nacional para la Gestión Integral de la Biodiversidad y sus Servicios Ecosistémicos. MADS.

Ministerio de la Protección Social de Colombia (2008). Vandemécun Colombiano de plantas medicinales. Imprenta Nacional de Colombia.

Morse, J. M. (1994). Emerging from the data: The cognitive processes of analysis in qualitative inquiry. En J. Morse (ed.), Critical issues in qualitative research methods, (pp. 23-46). Sage. https:// doi.org/10.2307/2076454

Ochoa, D. A. R. (2012). La salud pública como escenario para el diálogo de saberes. Revista CES Salud Pública, 3(1), 1-3.

Organización Mundial de la Salud [OMS]. (1988). Declaración de Chiang Mai, "Salvar vidas salvando las plantas". oms.

Organización Mundial de la Salud [OMS]. (2003). Directrices de la OMS sobre buenas prácticas agrícolas y de recolección (BPAR) de plantas medicinales. OMs. 
Pearce, D. W., \& Turner, P. K. (1995). Economía de los recursos naturales y del medioambiente. Celeste.

Programa de las Naciones Unidas para el Desarrollo [PNUD]. (2011). Mujeres rurales: gestoras de esperanza. PNUD

Reyes-Sánchez, G. L. (ed.). (2009). Diálogo de saberes: plantas medicinales, salud y cosmovisiones. Universidad Nacional de Colombia.

Rincón-Parra, N. S. (2012). Contexto y perspectiva de la red de suministro: Plantas aromáticas en Colombia. ECONÓMICAS CUC, 33(1), 135-156.

Rivera, A. P. T., Ortega-Cuadros, M., Melo-Ríos, A., \& Mier-Giraldo, H. J. (2017). Vigilancia tecnológica de plantas aromáticas: de la investigación a la consolidación de la agrocadena colombiana. Ciencia \& Tecnología Agropecuaria, 18(2), 353-377. https://doi.org/10.21930/rcta. vol18_num2_art:636

Romero, M. J., \& Rodríguez, E. B. (2010). Diálogo de saberes: Medicina tradicional y medicina occidental moderna. Revista de Investigaciones UNAD, 9(2), 125-133. https://doi. org/10.22490/25391887.676

Sandoval, R. J. C. (2018). Gestión intercultural de la biodiversidad: investigación acción en un proceso educativo con campesinos de Veracruz. Revista Interamericana de Educación de Adultos, 40(2), 119-135. https://www.redalyc.org/pdf/4575/ Resumenes/Resumen_457556293006_1.pdf

Sipahi, S., \& Mehpare, T. (2010). The analytic hierarchy process and analytic network process: an overview of applications. Management Decision, 48(5), 775-808. https://doi. org/10.1108/00251741011043920

Vera, M., \& Sánchez, S. M. (2015). Registro de algunas plantas medicinales cultivadas en San Cristóbal, municipio de Medellín (Antioquia, Colombia). Revista Facultad Nacional de Agronomía, 68(2), 7647-7658. https://doi. org/10.15446/rfnam.v68n2.50979 